\title{
Zur Verwendung der Partikel go in Modalverbkonstruktionen
}

\author{
Ruth von Rotz (Lugano)
}

\begin{abstract}
This article focusses on the use of the particle go in Swiss German modal verb constructions. The particle $g o$ is associated with the verb gaa 'go'. The research question is whether the particle go is solely used together with verbs entailing movement from one point to another. The presented results arise from questionnaire data and subsequent interviews. The area under investigation is situated in the highlands of the Canton St. Gallen in the East of Switzerland namely the villages Mels, Sargans, Wangs and Bad Ragaz.
\end{abstract}

\section{$1 \quad$ Einleitung}

Ziel der vorliegenden Untersuchung ist eine Darstellung der Verwendungsweise der eventuell auf das Verb gehen zurückgehenden Partikel $\mathrm{go}^{1}$ (Glaser 2003: 45) in Modalverbkonstruktionen. Die zwei Beispiele (1a) und (1b) sollen dieses Phänomen und dessen plausible Übersetzung ins Standarddeutsche veranschaulichen:

(1) a. Und ich muess ga mälchä. (Senti 1988: 347)

'Und ich muss melken gehen.'

b. Ewòtt gò ässe. (Fischer 1960: 358)

'Ich will essen gehen.'

Grammatisch ebenso korrekt ist eine Realisation der Beispiele (1a) und (1b) ohne die Partikel go. Analog dazu wird bei der Übersetzung ins Standarddeutsche der Infinitiv gehen weggelassen. Vgl. dazu die Beispiele (2a) und (2b):

(2) a. Und ich muess mälchä.

'Und ich muss melken.'

b. E wòtt ässe.

'Ich will essen.'

Zumindest für das Standarddeutsche kann hierbei vermerkt werden, dass zwischen den jeweiligen Varianten in (1) und (2) ein eindeutiger semantischer Unterschied besteht. In den Beispielen (1) bezeichnet der Infinitiv gehen nämlich den Aufbruch zu einer Tätigkeit (Brinkmann 1962: 283), die durch die Infinitive melken bzw. essen näher bestimmt ist. Die Beispiele (2) hingegen bezeichnen aufgrund des fehlenden Bewegungsverbs gehen keinen derartigen Aufbruch. Ob dies in gleichem Masse auch für das Schweizerdeutsche gilt, und ob die standarddeutsche Wiedergabe von go mit 'gehen' den semantischen und syntaktischen Eigenheiten der Partikel gerecht wird, ist allerdings zu bezweifeln. Den Anstoss zu dieser Vermutung geben semantische und syntaktische Ungereimtheiten, die sich bei einer konsequen-

1 Neben go existieren gemäss Id. (2, 322-326) auch die lautlichen Varianten ga, ge, gi und gu sowie reduplizierte Formen (z. B. goge). Zur Bezeichnung der Partikel wird in der vorliegenden Untersuchung durchgehend die Form go verwendet. Wo nicht auf Belege anderer Autoren zurückgegriffen wird, werden in Beispielen $g o$ und - wo für die Argumentation notwendig - die Varianten $g \ddot{a}$ und $g u$ verwendet. 
ten Wiedergabe von go mit 'gehen' ergeben. So wird man sich bei den folgenden beiden Belegen beim Übersetzen ins Standarddeutsche an der scheinbaren Doppelsetzung des Infinitivs gehen stören:

(3) a. Mer wend gān goge luegen. (Id. 2,324)

'Wir wollen gehen gehen schauen.'

b. 's Bettelfräuli wott go bettlen gōn. (Id. 2, 323)

'Die Bettelfrau [Diminutiv] will gehen betteln gehen.'

Weiter lassen auch bisherige Forschungsergebnisse vermuten, dass go nicht einfach mit 'gehen' wiedergegeben werden kann. So wurde beispielsweise im Rahmen des Nationalfondsprojekts "Dialektsyntax des Schweizerdeutschen" am Deutschen Seminar der Universität Zürich dem Problem der Verwendung von go in Modalverbkonstruktionen nachgegangen teilweise mit widersprüchlichen Resultaten, die einer genaueren Analyse bedürfen. ${ }^{2}$ An dieser Stelle sei auf eine Fragebogenaufgabe des genannten Projekts eingegangen, in der die Gewährspersonen verschiedene syntaktische Varianten auf ihre Plausibilität in einem gegebenen Kontext beurteilen sollten. Als Einleitung zu dieser Aufgabe wird eine Situation suggeriert, in der für die beschriebenen Akteure, einen Mann und eine Frau, eine Ortsveränderung im Sinne eines Gehens mit der Bedeutung "sich mit Hilfe der Füsse fortbewegen" (Helbig/Schenkel 1983: 232) ${ }^{3}$ nicht möglich sein sollte (s. Abb. 1). Trotz dieses Kontexts wurde von den beiden gegebenen Varianten, davon eine mit und eine ohne go, auch die Variante mit go überraschend häufig als eine in dieser Situation mögliche Antwort genannt. ${ }^{4}$ Was auf den ersten Blick wie freie Variation zwischen zwei verschiedenen syntaktischen Varianten aussieht, erweist sich bei genauerem Hinsehen jedoch wahrscheinlich als Trugschluss. Zieht man nämlich die zusätzlichen Kommentare der Gewährspersonen zu dieser Aufgabe hinzu, findet man, dass genau zu diesem Problem eine grosse Anzahl von Anmerkungen gemacht wurden, die besagen, dass zwischen den beiden Varianten mit und ohne die Partikel go sehr wohl ein semantischer Unterschied bestehe. Im Ganzen sind es 27 von total 31 zusätzlichen Bemerkungen, die festhalten, dass die Variante mit go eine Ortsveränderung bedinge und damit nicht zum angegebenen Kontext passe. Doch 27 zusätzliche Kommentare zu diesem Problem können noch keineswegs erklären, wie an 280 Orten Nennungen für die Variante mit go zustande kommen. Denkbar wäre, dass die Gewährspersonen den Kontext nicht richtig gelesen oder die vorgegebene Situation gedanklich um eine Fortbewegung der Personen ergänzt haben. Alternativ wäre, gerade wenn man sich die nicht mit Sicherheit geklärte Herkunft und Bedeutung von go vor Augen führt (vgl. Glaser 2003), eine Lesart von go abweichend vom Standarddeutschen 'gehen' in Betracht zu ziehen.

An den eben dargestellten Problemen setzt nun die vorliegende Untersuchung an. Es wird geklärt, ob lediglich in Formulierungen mit Modalverben, in denen eine Tätigkeit erst ausgeführt werden kann, indem oder nachdem eine Bewegung im Sinne eines Gehens stattfindet bzw. stattgefunden hat, eine Fügung mit der Partikel go auftritt. Es wird m. a. W. untersucht, ob der Gebrauch von go weitgehend dem Standarddeutschen 'gehen' entspricht oder ob die Partikel auch andere Funktionen übernehmen kann, die wiederum Auswirkungen auf die Semantik der gesamten Modalverbkonstruktion haben können.

2 Zum Projekt "Dialektsyntax des Schweizerdeutschen. Syntaktischer Atlas der Deutschen Schweiz (SADS)" siehe unter www.ds.uzh.ch/dialektsyntax/.

3 Fortan wird das standarddeutsche Verb gehen, falls nicht anderweitig spezifiziert, immer in dieser Bedeutung verwendet.

4 Es gab an 280 von 383 getesteten Orten jeweils mindestens eine Nennung für die Version Da mues i zersch go luege (vgl. Fussnote 2). 


\section{$2 \quad$ Methoden und Vorgehensweise}

\subsection{Wahl der Erhebungsmethode}

Um die eingangs umrissene Fragestellung zu beantworten, werden Sprachdaten aus einem kleinen Dialektgebiet, das die Dörfer Mels, Sargans, Wangs und Bad Ragaz im Sarganserland (St. Galler Oberland) umfasst, analysiert. Um eine effiziente Vorgehensweise zu garantieren und gleichzeitig aussagekräftige Resultate zu gewinnen, werden die Daten direkt mündlich mithilfe eines eigens für die vorliegende Untersuchung zusammengestellten Fragebogens erhoben. Die aus dieser Methode resultierenden Nachteile, die oft in der Beeinflussung der Gewährspersonen und in der in einem gewissen Sinne künstlichen Interviewsituation gesehen werden (Glaser 2000: 259), werden durch entscheidende Vorteile relativiert. Diese bestehen darin, dass durch die direkte Befragung nicht nur das Vorkommen eines bestimmten sprachlichen Phänomens getestet, sondern auch Erklärungen für die Verwendung oder Ablehnung dieser Erscheinung gefunden werden können (Cornips/Poletto 2005: 942). Ausserdem besteht bei mündlicher Abfragung die Möglichkeit, auf die Antworten der Gewährspersonen unmittelbar zu reagieren und rückzufragen, warum eine bestimmte sprachliche Erscheinung auftaucht oder abgelehnt wird. Solche Rückfragen wurden für die vorliegende Untersuchung gezielt eingesetzt, da nicht nur die syntaktische Akzeptabilität von Strukturen generell, sondern in erster Linie deren diffizile Unterschiede in Bezug auf die Semantik getestet werden.

\subsection{Ausgestaltung des Fragebogens}

Das Format des für die Erhebung verwendeten Fragetyps orientiert sich am Aufbau der in der Einleitung diskutierten Aufgabe des SADS-Projekts. Die Aufgaben werden jeweils durch eine Situationsbeschreibung eingeleitet (Glaser 2000: 273). Diese gibt den Kontext vor und soll den Gewährspersonen das Gefühl geben, sich in einer alltäglichen Kommunikationssituation zu befinden (vgl. Bucheli/Glaser 2002). Daraufhin folgt die Angabe der Varianten im Dialekt. Diese Varianten können inhaltlich als Reaktion auf die durch den Kontext angedeutete Situation charakterisiert werden. Anschliessend folgt die Frage nach der natürlichsten Variante sowie nach allfälligen weiteren möglichen Varianten (vgl. Abb. 1):

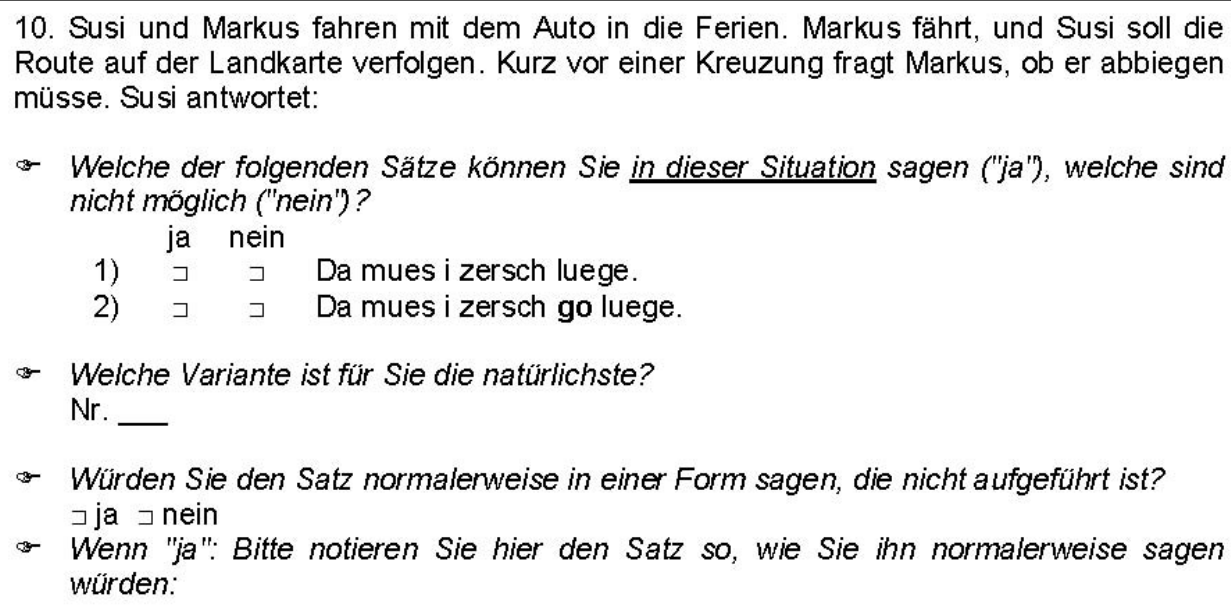

Welche der folgenden Sätze können Sie in dieser Situation sagen ("ja"), welche sind nicht möglich ("nein")?

1) ja $\quad \begin{gathered}\text { nein } \\ \lrcorner\end{gathered} \quad$ Da mues i zersch luege.

2) $\lrcorner \quad \sqsupset \quad$ Da mues i zersch go luege.

Welche Variante ist für Sie die natürlichste? $\mathrm{Nr}$.

Würden Sie den Satz normalenweise in einer Form sagen, die nicht aufgeführt ist? $\sqsupset$ ja $\lrcorner$ nein

- Wenn "ja": Bitte notieren Sie hier den Satz so, wie Sie ihn normalerweise sagen würden:

Abb. 1: Aufgabe 10 des vierten Fragebogens (IV.10) des SADS-Projekts

Der für die vorliegende Untersuchung verwendete Fragebogen besteht aus 12 solcher Aufgaben, in denen die sechs Modalverben chünä 'können', mü̈̈gä 'mögen', törfä 'dürfen', müäs ä 
'müssen', sölä 'sollen' und welä 'wollen'5 je zweimal den Infinitiv eines Verbs als Ergänzung nehmen, und zwar jeweils in einer Situation, die zu der durch den Infinitiv ausgedrückten Tätigkeit eine Bewegung im Sinne eines in der zur Tätigkeitsausübung keine solche

Bewegung gefordert ist. ${ }^{6}$ Als Reaktion auf die suggerierte Sprechsituation stehen in jeder Aufgabe drei Varianten (a), (b) und (c) zur Verfügung. Das Muster dieser Varianten sieht dabei immer folgendermassen aus:

a. Modalverb + Infinitiv

b. Modalverb $+g o+$ Infinitiv

c. Modalverb $+g o+$ Infinitiv + goo

Dabei können zwischen Modalverb und Infinitiv bzw. zwischen Modalverb und go nominale, präpositionale und adverbiale Ergänzungen stehen. Im Unterschied zum SADS-Projekt, in dem bei der Aufgabe IV.10 (s. Abb. 1) nur die Varianten nach dem Muster von (a) und (b) abgefragt werden, soll in dieser Untersuchung auch die Variante (c) untersucht werden, da diese nachgewiesenermassen ebenfalls existiert, ${ }^{7}$ ohne dass ihre Verwendungsweise, besonders auch im Unterschied zu (2), genauer bekannt ist. ${ }^{8}$

\subsection{Wahl der Gewährspersonen und Erhebung der Daten}

Die für dieses Projekt ausgewählten Gewährspersonen (GP) sind alle im Sarganserland geboren und aufgewachsen, stammen aus Familien, die seit mindestens zwei Generationen in diesem Gebiet ansässig sind, sprechen den lokalen Dialekt und sollten aufgrund ihrer Dialektkompetenz in der Lage sein, die ihnen gestellten Aufgaben problemlos zu lösen. Mit Ausnahme von zwei Personen, die nur ihre ersten 22 Lebensjahre im Sarganserland verbrachten, ${ }^{9}$ wohnen ausserdem alle GP seit jeher in dieser Region. Um herauszufinden, ob es Unterschiede im Gebrauch der Partikel go zwischen Altersgruppen gibt, wurden die Aufgaben aus dem Fragebogen den fünf jüngeren (unter 30 Jahren) und fünf älteren (über 30 Jahren) GP im Interview vorgelegt. Die Auswahl der GP nach Alter, Geschlecht und Wohnort sieht wie folgt aus:

5 Diese Verben erfüllen alle der folgenden Kriterien (Eisenberg 2004: 99-107): 1. Sie nehmen, wenn nicht als Vollverben gebraucht, einen oder zwei reine Infinitive, also Infinitive ohne zu, als Ergänzung. 2. Sie haben keinen Imperativ. 3. Sie bilden, sofern nicht als Vollverben gebraucht, die zusammengesetzten Formen der Vergangenheit (Perfekt und Plusquamperfekt) häufig nicht mit dem Partizip II, sondern mit dem Infinitiv Präsens. 4. Sie sind ehemalige Präteritopräsentien, d. h. sie bilden das Präsens so wie die starken Verben das Präteritum (Die 1. P. Sg. und die 3. P. Sg. stimmen formal überein und sind überdies endungslos).

6 Der verwendete Fragebogen kann im Anhang eingesehen werden.

7 Belege für die Existenz der Variante (c) sind die Beispiele (3).

8 Die konsultierte Literatur beschränkt sich darauf, auf das Vorkommen der beiden Varianten (b) und (c) hinzuweisen, ohne allfällige Auftretensbedingungen für die eine oder die andere Variante zu nennen (vgl. z. B. Christen 1990, Baur 2002).

9 Sollten im Laufe der Erhebung Fremdeinflüsse auf den ursprünglichen Dialekt dieser Gewährspersonen festgestellt werden, wird diesen in der Auswertung der Resultate Rechnung getragen. Es ist jedoch anzunehmen, dass solche Einflüsse gering oder gar nicht festzustellen sind, da die Gewährspersonen immer noch einen ausgeprägten Sarganserländer Dialekt sprechen. 


\begin{tabular}{|c|c|c|c|}
\hline \multicolumn{2}{|c|}{ Jüngere GP } & \multicolumn{2}{|c|}{ Ältere GP } \\
\hline GP 1: & 15, w, Sargans & GP 6: & 49, w, Sargans \\
\hline GP 2: & 22, w, Mels & GP 7: & $49, \mathrm{~m}$, Sargans \\
\hline GP 3: & 23, w, Bad Ragaz & GP 8: & $56, \mathrm{w}$, Wangs \\
\hline GP 4: & 26, w, Uster & GP 9: & 57, w, Mels \\
\hline GP 5: & 28, w, Zürich & GP 10: & $59, \mathrm{w}$, Wangs \\
\hline
\end{tabular}

Mit den Antworten der GP wird folgendermassen verfahren: Als erstes wird erfasst, welche der gegebenen Varianten eine GP in Bezug auf den gegebenen Kontext als möglich betrachtet. ${ }^{10}$ Eine Variante wird nur dann als zur Situation passend und damit als möglich gewertet, wenn die GP die vorgegebene Situationsbeschreibung nicht verändert. Werden zusätzliche Annahmen getroffen, werden diese diskutiert und in die Bewertung unter Angabe dieser Annahmen mit einbezogen. Dann sollen die GP von den für sie möglichen Varianten die natürlichste nennen. Schliesslich folgt unter den Stichwörtern "Weitere Variante/Kommentar" die Aufforderung, allfällige zusätzlich bestehende Varianten $\mathrm{zu}$ nennen. Wo es für die Untersuchung aufschlussreich erscheint, werden an dieser Stelle die GP ausserdem gebeten, sämtliche Varianten, ob in der gegebenen Situation möglich oder unmöglich, semantisch voneinander abzugrenzen. Hinter dem beschriebenen Verfahren steckt folgende Überlegung: Werden Fügungen mit Modalverb + go in Situationen, in denen zur eigentlichen Tätigkeitsausübung (ausgedrückt durch einen Infinitiv) keine Bewegung im Sinne eines Gehens stattfinden kann oder muss, nicht verwendet, in Fällen mit zur Tätigkeitsausübung (ausgedrückt durch denselben Infinitiv) notwendiger Bewegung im Sinne eines Gehens hingegen schon, ist anzunehmen, dass go auf das Verb 'gehen' hinweist. Werden hingegen in Situationen, die ein Gehen nicht erlauben oder ermöglichen, Varianten mit go verwendet, ist zu untersuchen, welche Funktionen die Partikel in den entsprechenden Fällen übernimmt und welche Auswirkungen sich auf die Semantik der Gesamtkonstruktion im einzelnen Fall ergeben.

\section{Ergebnisse}

Zunächst einmal ist festzuhalten, dass, mit Ausnahme von wenigen Einzelfällen, auf die in den folgenden Unterabschnitten ausdrücklich verwiesen wird, die Varianten (a), (b) und (c) von den GP als grundsätzlich in ihrem Dialekt verwendete syntaktisch mögliche Varianten beurteilt werden. Ausserdem gilt für alle Resultate, falls nicht im Einzelfall anderweitig spezifiziert, dass die Variante (a) als Formulierung, die keine Bewegung im Sinne eines Gehens verlangt oder zulässt, beurteilt wird. Die Varianten (b) und (c) werden hingegen, wiederum falls nicht anderweitig dargestellt, als semantisch und syntaktisch gleichwertige Formulierungen gesehen, die eine derartige Bewegung mit einschliessen. Tabelle 1 fasst die Resultate der Untersuchung zusammen. ${ }^{11}$ Die Untersuchung zeigt jedoch, dass es zwischen dieser groben Unterscheidung noch sehr viel feinere semantische Nuancen gibt, die ausserdem stark subjektiv gefärbt sind und ihrerseits wiederum Rückschlüsse auf die Funktion der

10 Werden Varianten aus syntaktischen Gründen nicht akzeptiert, werden die Formulierungen "syntaktisch nicht möglich/syntaktisch unmöglich" oder "syntaktisch nicht akzeptiert" verwendet. Wird hingegen eine Variante zwar als syntaktisch möglich, jedoch nicht zum Kontext passend empfunden, wird dies lediglich mit den Formulierungen "in dieser Situation nicht möglich/in dieser Situation unmöglich" oder "in dieser Situation nicht akzeptiert" bzw. schlicht "nicht möglich/unmöglich" oder "nicht akzeptiert" gekennzeichnet. An dieser Stelle wird in den Befragungen ausserdem noch einmal klar darauf hingewiesen, dass diejenigen Varianten genannt werden sollen, die im Dialekt der Gewährspersonen tatsächlich verwendet werden (die man "hört"), denn Untersuchungen haben ergeben, dass sich Akzeptabilität und tatsächliche Verwendung syntaktischer Konstruktionen nicht notwendigerweise decken müssen (vgl. Cornips/Poletto 2005).

${ }^{11}$ In der Folge werden in Tabellen die folgenden Abkürzungen verwendet: M für Modalverb, I für Infinitiv, "mögl." für mögliche Varianten und "nat." für natürlichste Variante. 
verschiedenen Varianten erlauben. Auf diese Unterschiede wird, wo angebracht, in den folgenden Unterabschnitten im Detail eingegangen.

\begin{tabular}{|c|c|c|c|c|c|}
\hline Modalverb & Infinitiv & Bewegung & (a) & (b) & (c) \\
\hline 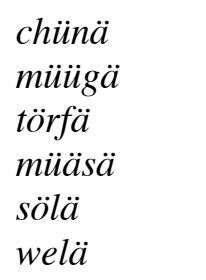 & $\begin{array}{l}\text { hälfä } \\
\text { probiärä } \\
\text { spiiläa } \\
\text { machä } \\
\text { vorläsä } \\
\text { schloufä }\end{array}$ & $\begin{array}{l}\text { nein } \\
\text { nein } \\
\text { nein } \\
\text { nein } \\
\text { nein } \\
\text { nein }\end{array}$ & $\begin{array}{r}10 \\
10 \\
10 \\
10 \\
10 \\
9\end{array}$ & $\begin{array}{l}- \\
- \\
1 \\
- \\
- \\
6\end{array}$ & $\begin{array}{l}- \\
- \\
1 \\
- \\
\overline{7}\end{array}$ \\
\hline 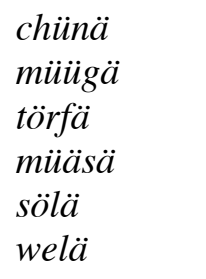 & $\begin{array}{l}\text { hälfä } \\
\text { probiärä } \\
\text { spiiläa } \\
\text { machä } \\
\text { vorläsä } \\
\text { schloufä }\end{array}$ & $\begin{array}{l}\text { ja } \\
\text { ja } \\
\text { ja } \\
\text { ja } \\
\text { ja } \\
\text { ja }\end{array}$ & $\begin{array}{l}4 \\
1 \\
4 \\
6 \\
4 \\
3\end{array}$ & $\begin{array}{r}8 \\
10 \\
10 \\
10 \\
10 \\
8\end{array}$ & $\begin{array}{r}10 \\
4 \\
7 \\
5 \\
7 \\
9\end{array}$ \\
\hline
\end{tabular}

Tab. 1: Zusammenfassung der als möglich angegebenen Varianten

(a) $=\mathbf{M}+\mathbf{I} ;$ (b) $=\mathbf{M}+$ go $+\mathbf{i} ;(\mathbf{c})=\mathbf{M}+\mathbf{g o}+\mathbf{I}+$ goo

\subsection{Situationen ohne Bewegung im Sinne eines Gehens}

Die Resultate der Aufgaben, in denen die Situationsbeschreibung keine Bewegung im Sinne eines Gehens vorsieht, sind, wie Tabelle 2 zeigt, recht eindeutig: Mit Ausnahme der Kombinationen törfä und spiilä sowie welä und schloufä wird in allen Aufgaben (a) als einzig mögliche und damit von den gegebenen Konstruktionen auch als natürlichste Variante angesehen. Begründet wird diese Wahl mit der Unmöglichkeit oder Unnötigkeit eines Gehens in den dargestellten Situationen. Einer besonderen Beschreibung bedürfen damit lediglich die Resultate der ersten und der dritten Aufgabe (Kombinationen törfä und spiilä sowie welä und schloufä).

\begin{tabular}{|c|c|c|c|c|c|c|c|}
\hline \multirow{2}{*}{ Modalverb } & \multirow{2}{*}{ Infinitiv } & \multicolumn{2}{|c|}{ (a) } & \multicolumn{2}{|c|}{ (b) } & \multicolumn{2}{|c|}{ (c) } \\
\hline & & mögl. & nat. & mögl. & nat. & mögl. & nat. \\
\hline chünä & hälfä & 10 & 10 & - & - & - & - \\
\hline mü̈̈̈g̈ & probiärä & 10 & 10 & - & - & - & - \\
\hline törfä & spiilä & 10 & 10 & 1 & - & 1 & - \\
\hline müäsä & machä & 10 & 10 & - & - & - & - \\
\hline sölä & vorläsä & 10 & 10 & - & - & - & - \\
\hline welä & schloufä & 9 & 7 & 6 & - & 7 & 2 \\
\hline
\end{tabular}

Tab. 2: Zusammenfassung der möglichen und natürlichen Varianten in den Aufgaben ohne Bewegung im Sinne eines Gehens. (a) $=\mathbf{M}+\mathbf{I} ;(\mathbf{b})=\mathbf{M}+\mathbf{g o}+\mathbf{i} ;(\mathbf{c})=\mathbf{M}+\mathbf{g o}+\mathbf{I}+\mathbf{g o o}$

Bei der ersten Aufgabe beurteilt nur eine ältere GP die Varianten (b) und (c) als möglich. Sie begründet diese Wahl folgendermassen: In der beschriebenen Situation sei es denkbar, dass sich der Sprecher, allerdings ohne zu gehen, vor dem Spielen zurechtrücken und dem Spielpartner zuwenden müsse. In diesem Sinne bezeichnen die Varianten mit go folglich nicht ein Gehen, sondern lediglich ein Anschicken zu einer Tätigkeit, das eine Bewegung ohne zu gehen verlangt. Als Einzelnennung wäre diese Beobachtung in einer grösser angelegten Untersuchung auf ihre Richtigkeit hin zu überprüfen.

Bei der dritten Aufgabe präsentieren sich die Resultate wohl aufgrund des etwas offeneren Kontexts anders als bei den anderen Aufgaben dieser Art. Die grösste Akzeptanz findet aber auch in dieser Situation die Variante (a) mit neun Nennungen, gefolgt von Variante (c) mit sieben und Variante (b) mit sechs Nennungen. Ausserdem stellt Variante (a) mit sieben 
Stimmen auch für die Mehrheit der GP die natürlichste Variante dar. Alle zehn GP geben mit dem Verweis auf die Unnötigkeit eines Gehens in der dargestellten Situation an, dass der Sprecher mit der Äusserung von Variante (a) den Angesprochenen auffordere, unmittelbar neben ihm auf demselben Sofa zu schlafen. Lediglich eine jüngere GP wendet ein, dass eine solche Aufforderung in der gegebenen Situation nicht denkbar sei und wertet deshalb (a) nicht als mögliche Variante.

Die Varianten mit go werden ausschliesslich von denjenigen GP, die sich (auch) vorstellen können, dass der Sprecher den Angesprochenen auffordert, das Zimmer zu verlassen, als mögliche Reaktionen betrachtet. Zwei ältere GP geben an, die Formulierung der Frage nach dem Muster von (b) lasse offen, ob der Angesprochene am selben Ort wie der Sprecher oder in einem anderen Zimmer schlafen wolle. In dieser Verwendung verweist go folglich nicht zwingend auf das Standarddeutsche 'gehen', sondern könnte Unsicherheit oder Unentschiedenheit anzeigen. Mit der Begründung, der Kontext der Aufgabe sei offen und unsicher, wertet eine GP die Konstruktion (b) ausserdem als natürlichste Variante.

Eine ältere GP erwähnt in der gegebenen Situation, dass Variante (c) gegenüber Variante (b) eine grössere räumliche und zeitliche Distanz zwischen Vorschlag (oder Aufforderung) und tatsächlicher Ausführung suggeriere. Auf eine andere Art grenzen ausserdem eine jüngere und eine ältere die beiden Varianten mit go ab: Die jüngere GP empfindet (c) als fragend und höflicher, während die ältere GP diese Variante als sehr bestimmte und überdies eindringlichere Reaktion empfindet. Beide nennen (c) ferner als in dieser Situation natürlichste Variante.

Aus den Interviews geht damit ganz klar hervor, dass die Nennungen für die natürlichste Variante auf die jeweiligen Annahmen der GP zur Intention des Sprechers verweisen: Nimmt die GP an, der Sprecher wolle den Angesprochenen nicht wegschicken, nennt sie (a) als natürlichste Variante. Geht sie hingegen davon aus, der Angesprochene solle den Raum wechseln, kommen die Varianten mit go zum Zug, die äusserst unterschiedlich empfunden werden und aufgrund zusätzlich getroffener Annahmen zum gegebenen Kontext als natürlichste Varianten genannt werden.

\subsection{Situationen mit Bewegung im Sinne eines Gehens}

Die Resultate der Aufgaben, in denen die Situationsbeschreibung eine Bewegung im Sinne eines Gehens vorsieht, nehmen sich von Aufgabe zu Aufgabe sehr unterschiedlich aus. In der Folge soll deshalb jede dieser Aufgaben im Detail besprochen werden.

\subsubsection{Chünä in Verbindung mit dem Infinitiv hälfä (2. Aufgabe)}

Bei dieser Aufgabe wird die Möglichkeit eines Gehens von allen GP erwähnt. So werden denn auch die Varianten (b) und (c) in dieser Situation mehrheitlich akzeptiert (s. Tab. 3).

\begin{tabular}{|l|r|r|r|}
\hline & (a) $\mathbf{M}+\mathbf{I}$ & (b) $\mathbf{M}+\boldsymbol{g o}+\mathbf{I}$ & (c) $\mathbf{M}+\boldsymbol{g o}+\mathbf{I}+\mathbf{g o o}$ \\
\hline möglich & 4 & 8 & 10 \\
natürlich & 1 & 2 & 7 \\
\hline
\end{tabular}

Tab. 3: Resultate der 2. Aufgabe (chünä ergänzt durch hälfä)

Zwei ältere GP werten in der gegebenen Situation Variante (b) als unmöglich; diese könne nur verwendet werden, wenn sich die angesprochene Person z. B. im Nebenzimmer befinde. Da die Kommunikation aber per Telefon stattfinde, sei anzunehmen, dass zwischen Sprecher und Angesprochenem eine grössere räumliche Distanz liege, welcher nur durch Verwendung von Variante (c) Rechnung getragen werden könne. 
Für zwei jüngere und zwei ältere GP ist im gegebenen Kontext auch eine Verwendung von Variante (a) denkbar. Sie signalisiere, dass Hilfe jeder Art erwünscht sei. Damit lasse sie offen, ob die Angesprochene helfe, indem sie zu der unterstützungsbedürftigen Person hingehe oder indem sie zu Hause bleibe (z. B. durch Unterstützung beim Kinderhüten bei sich zu Hause). Eine ältere GP gibt (a) aufgrund dieser verschiedenen Möglichkeiten, die sie impliziere, als natürlichste Variante an. Für die anderen drei GP, die (a) als möglich beurteilen, ist der Gebrauch dieser Variante hingegen lediglich möglich, aber in der gegebenen Situation wenig wahrscheinlich.

Die weitere Verteilung der Angaben der natürlichsten Variante lässt sich folgendermassen erklären: Zwei jüngere GP favorisieren Variante (b), da sie diese als weniger umständlich empfinden als (c). Die restlichen sieben GP geben (c) als natürlichste Variante an, da sie diese als höflicher oder fragender empfinden als (b) und in der gegebenen Situation zu erwarten sei, dass der Sprecher seine Bitte höflich vortrage. Die Verteilung der Nennungen für die natürlichste Variante basiert damit zumindest teilweise auf zusätzlich zum gegebenen Kontext getroffenen Annahmen.

\subsubsection{Mü̈̈gä in Verbindung mit dem Infinitiv probiärä (12. Aufgabe)}

Bei dieser Aufgabe wird die Notwendigkeit eines Gehens von allen zehn GP zur Begründung der Wahl von (b) herangezogen. Eine jüngere GP betrachtet den Gebrauch von (a) als möglich (und gleichzeitig als am natürlichsten), da es in der dargestellten Situation auch ohne explizite Äusserung von go klar sei, dass die Kinder nach draussen gehen müssen (s. Tab. 4). Go verweist für diese GP damit klar auf 'gehen'. Auffällig ist die im Vergleich mit den andern Aufgaben dieses Typs geringe Akzeptanz der Variante (c). Diese wird lediglich von einer jüngeren und drei älteren GP als mögliche Variante betrachtet.

\begin{tabular}{|l|r|r|r|}
\hline & (a) $\mathbf{M}+\mathbf{I}$ & (b) $\mathbf{M}+\boldsymbol{g o}+\mathbf{I}$ & (c) $\mathbf{M}+\boldsymbol{g o}+\mathbf{I}+\mathbf{g o o}$ \\
\hline möglich & 1 & 10 & 4 \\
natürlich & 1 & 6 & 3 \\
\hline
\end{tabular}

Tab. 4: Resultate der 12. Aufgabe (müügä ergänzt durch probiärä)

Die restlichen vier jüngeren GP beurteilen die Variante (c) im vorliegenden Fall als syntaktisch unmöglich. Was die natürlichste Variante betrifft, sprechen sich lediglich drei ältere GP für (c) aus. Eine dieser GP begründet dies mit der grösseren räumlichen Distanz zwischen Küche und Garten, die (c) im Unterschied zu (b) bedinge und die im vorliegenden Fall zu erwarten sei. Die anderen zwei älteren GP hingegen erklären die Angabe von (c) als natürlichste Variante mit dem Hinweis darauf, dass (c) gegenüber (b) grammatisch korrekter sei und im vorliegenden Fall, in dem ein Vater in Vorbildfunktion zu seinen Kindern spreche, die grammatisch korrektere Variante, also (c), zu erwarten sei. Die jüngere GP, die (c) als möglich angibt, sieht diese als etwas umständlich (wenn auch syntaktisch möglich) und damit nicht als natürlichste Variante an. Zwei ältere GP nennen die kleinere Distanz, die die Verwendung von (b) im Gegensatz zu (c) ausdrücke und die im dargestellten Fall zu erwarten sei, als ausschlaggebend für ihre Wahl von (b) als natürlichste Variante. Damit ist die Verteilung der Nennungen für die natürlichste Variante teilweise syntaktisch und teilweise semantisch bedingt.

\subsubsection{Törfä in Verbindung mit dem Infinitiv spiilä (8. Aufgabe)}

Auch bei dieser Aufgabe wird die Notwendigkeit eines Gehens von allen GP klar erkannt und als ausschlaggebend für die Wahl der Varianten (b) und (c) angegeben: Variante (b) erachten alle zehn GP und Variante (c) deren sieben als möglich (s. Tab. 5): 


\begin{tabular}{|l|r|r|r|}
\hline & (a) $\mathbf{M}+\mathbf{I}$ & (b) $\mathbf{M}+\mathbf{g o}+\mathbf{I}$ & (c) $\mathbf{M}+\mathbf{g o}+\mathbf{I}+$ goo \\
\hline möglich & 4 & 10 & 7 \\
natürlich & 1 & 6 & 3 \\
\hline
\end{tabular}

Tab. 5: Resultate der 8. Aufgabe (törfä ergänzt durch spiilä)

Diejenigen drei, die (c) in der gegebenen Situation nicht akzeptieren, tun dies aus folgenden Gründen: Für zwei jüngere GP ist diese Variante schlicht syntaktisch unmöglich, für eine ältere GP hingegen wird mittels der Formulierung nach dem Muster von (c) eine grosse Distanz ausgedrückt, die für das in der Situationsbeschreibung vorgestellte Kind noch nicht allein zu bewältigen sei. Diese drei GP geben deshalb mit der Begründung, ein Gehen sei in dieser Situation absolut nötig, (b) als natürlichste Variante an.

Eine ältere und drei jüngere GP betonen vor allem die zusätzliche zeitliche Komponente, die Variante (c) im Gegensatz zu (b) beinhalte: Während das Kind bei Äusserung von Variante (b) sofort darauf dränge, hinauszugehen, nehme es bei der Frage mit der Struktur Modalverb + go + Infinitiv + goo eher in Kauf, eine Verrichtung noch zu Ende zu führen, bevor es hinausgehe. Durch Übertragung dieser Annahmen auf die gegebene Situation sind die Nennungen für die natürlichste Variante zu erklären: Eine jüngere GP wertet (c), zwei jüngere und eine ältere GP nennen jedoch (b) als natürlichste Variante. Zwei ältere GP hingegen empfinden eine Äusserung nach dem Vorbild von Variante (c) gegenüber (b) als dringender und bestimmter und ausserdem als in der gegebenen Situation natürlichste Variante.

Drei jüngere und eine ältere GP erachten auch die Variante (a) ohne go als mögliche Frage, wobei sie folgendermassen argumentieren: Aufgrund des Kontextes sei ohnehin klar, dass das Kind hinausgehen müsse, deshalb käme eine Verwendung von go einem Pleonasmus gleich. Folglich verweist für diese GP go in der dargestellten Verwendung klar auf 'gehen'. Allerdings wertet lediglich die ältere dieser GP (a) als natürlichste Variante. Die Nennungen für die natürlichste Variante sind damit nur teilweise syntaktisch bedingt und spiegeln hauptsächlich die getroffenen Annahmen wider, anhand derer die gegebene Situation beurteilt wird.

\subsubsection{Müäsä in Verbindung mit dem Infinitiv machä (5. Aufgabe)}

Auch bei dieser Aufgabe wird wiederum mit dem Verweis auf die Notwendigkeit eines Gehens im gegebenen Kontext die Variante (b) von allen GP als möglich beurteilt. Variante (c) hingegen wird von drei jüngeren GP aus syntaktischen, von zwei älteren jedoch aus semantischen Gründen abgelehnt: Die Formulierung mit go + Infinitiv + goo bedinge für dieses die Unmittelbarkeit einer Handlung thematisierende Beispiel eine zu grosse zeitliche Distanz zwischen ausgeführter und auszuführender Handlung.

\begin{tabular}{|l|r|r|r|}
\hline & (a) $\mathbf{M}+\mathbf{I}$ & (b) $\mathbf{M}+$ go + I & (c) $\mathbf{M}+$ go + I + goo \\
\hline möglich & 6 & 10 & 5 \\
natürlich & 3 & 5 & 2 \\
\hline
\end{tabular}

Tab. 6: Resultate der 5. Aufgabe (müäsä ergänzt durch machä)

Zwei andere ältere GP schreiben der Variante (c) in Bezug auf die auszuführende Tätigkeit ebenfalls eine grössere zeitliche Verzögerung als (b) zu, argumentieren aber, dass (c) den Widerwillen, den das Kind in der einleitenden Situationsbeschreibung zeige, am besten reflektiere und folglich die in dieser Situation natürlichste Variante sein müsse. Die anderen zwei jüngeren und eine ältere GP, die (c) ebenfalls als möglich einstufen, werten (b) allerdings als strukturell einfachere und gleichzeitig natürlichste Variante. 
Eine ältere und fünf jüngere GP beurteilen auch die Variante ohne go als möglich, da aufgrund der beschriebenen Situation ein Gehen ohnehin notwendig sei und damit nicht zwingend zusätzlich ausgedrückt werden müsse. Für zwei jüngere und die eine ältere GP stellt diese syntaktisch einfachste aber nicht minder klare Frage gleichzeitig die natürlichste Variante dar. Die restlichen drei jüngeren GP, die (a) als möglich einstufen, bewerten allerdings Variante (b) gegenüber (a) als natürlichere und damit gleichzeitig als natürlichste Variante, da sie (c) als syntaktisch unmöglich erachten. Vier GP hingegen geben an, die Verwendung von (a) würde bedeuten, dass das Kind sich ohne aufzustehen der auszuführenden Tätigkeit widmete. Diese GP sehen deshalb (b) oder (c), die beide go im Sinne von 'gehen' beinhalten, als natürlichste Variante: Zwei entscheiden sich, wie oben bereits dargestellt, für (c) und die anderen zwei geben (b) als einzige und damit gleichzeitig natürlichste Variante an.

\subsubsection{Sölä in Verbindung mit dem Infinitiv vorläsä (9. Aufgabe)}

Auch bei dieser Aufgabe geben alle zehn GP die Variante (b) und sieben GP die Variante (c) als möglich an, da der Sprecher in der dargestellten Situation mit grosser Wahrscheinlichkeit ein Gehen vorsehe (s. Tab. 7). Eine jüngere GP hingegen beurteilt (c) als syntaktisch nicht möglich und eine jüngere und eine ältere GP geben an, (c) sei aufgrund der grossen räumlichen und zeitlichen Distanz, die sie zwischen der Äusserung der Bemerkung und der intendierten Handlung zulasse, in der dargestellten Situation, die unmittelbares Handeln erfordere, nicht angebracht.

\begin{tabular}{|l|r|r|r|}
\hline & (a) $\mathbf{M}+\mathbf{I}$ & (b) $\mathbf{M}+\boldsymbol{g o}+\mathbf{I}$ & (c) $\mathbf{M}+$ go + I + goo \\
\hline möglich & 4 & 10 & 7 \\
natürlich & - & 6 & 4 \\
\hline
\end{tabular}

Tab. 7: Resultate der 9. Aufgabe (sölä ergänzt durch vorläsä)

Vier GP könnten sich auch eine Verwendung von Variante (a) vorstellen. Eine jüngere GP begründet diese Wahl damit, dass man die Intention des Sprechers auch ohne Verwendung der Partikel go richtig deute, da man im vorliegenden Fall annehmen könne, dass der Sprecher ohnehin vorhabe, den Raum zu verlassen (d. h. zu gehen) und nach den Kindern zu sehen. Zwei ältere und eine jüngere GP bewerten Variante (a) in der gegebenen Situation ebenfalls als möglich. Allerdings frage der Sprecher damit nicht mehr, ob ein Bedürfnis bestehe (nämlich die Kinder zu beruhigen), sondern sehe dieses als gegeben an und stelle dafür das Mittel (ausgedrückt durch ä Gschichtli), mit dem er sein Ziel erreichen wolle, in Frage.

In Bezug auf die natürlichste Variante entscheiden sich jedoch alle GP für eine der beiden Varianten mit der Partikel go. Alle jüngeren GP geben (b) als natürlichste Variante an, da diese weniger umständlich und überdies gebräuchlicher sei als (c). Ausserdem sieht auch eine ältere GP, die weder (a) noch (c) in dieser Situation für mögliche Reaktionen hält, in (b) die natürlichste Variante. Die restlichen vier älteren GP wählen aufgrund der "grammatischen Korrektheit" und "Vollständigkeit des Satzes", die vom Sprecher im gegebenen Kontext zu erwarten seien, (c) als natürlichste Variante.

\subsubsection{Welä in Verbindung mit dem Infinitiv schloufä (11. Aufgabe)}

Bei dieser Aufgabe geben alle GP mit der Begründung, eine Bewegung im Sinne eines Gehens sei in der skizzierten Situation wohl vorgesehen, (b), (c) oder (b) und (c) zusammen als mögliche Varianten an. Bloss eine jüngere GP bewertet Variante (c) als syntaktisch nicht möglich (s. Tab. 8). 


\begin{tabular}{|l|r|r|r|}
\hline & (a) $\mathbf{M}+\mathbf{I}$ & (b) $\mathbf{M}+$ go + I & (c) $\mathbf{M}+$ + go + I + goo \\
\hline möglich & 3 & 8 & 9 \\
natürlich & - & 1 & 9 \\
\hline
\end{tabular}

Tab. 8: Resultate der 11. Aufgabe (welä ergänzt durch schloufä)

Für drei GP käme ausserdem auch eine Verwendung von Variante (a) in Frage. In dieser Verwendung käme die Äusserung allerdings nicht mehr einer Frage nach der Neigung des Angesprochenen bzw. einem Vorschlag gleich, sondern wäre lediglich eine rhetorische Frage, mit der der Sprecher seine Meinung kundtäte, d. h. die Party als langweilig betitelte. Keine der drei GP wertet diese Möglichkeit allerdings als natürlichste Variante.

Zum Unterschied zwischen (b) und (c) äussern sich die GP unterschiedlich: Drei jüngere und drei ältere GP betonen die grössere räumliche und zeitliche Distanz, die es bei (c) zwischen der Äusserung des Vorschlags und dem angestrebten Ziel (ausgedrückt durch den Infinitiv schloufä) zu überwinden gelte. Zwei dieser GP werten Variante (b) explizit nur als Gang ins Nebenzimmer und akzeptieren sie in der vorgegebenen Situation nicht als mögliche Variante, da sie annähmen, Sprecher und Angesprochener hätten noch eine längere Heimfahrt vor sich. Als einzig mögliche und damit natürlichste Variante bewerten diese zwei GP die Variante (c). Die anderen vier GP, die (b) und (c) bezüglich räumlicher und zeitlicher Dimension ebenfalls unterschiedlich bewerten, schliessen einen durch Variante (b) implizierten kürzeren Weg (z. B. ins Nebenzimmer oder ins Nachbarhaus) im gegebenen Kontext zwar nicht aus, sehen allerdings (c) aufgrund der Annahme, Sprecher und Angesprochener hätten noch einen längeren Weg vor sich, als natürlichste Variante an. Eine jüngere GP sieht (c) hingegen als höflichere Art zu fragen an als (b) und wertet aufgrund der Vermutung, der Sprecher stelle seine Frage auf eine freundliche und höfliche Art, (c) als natürlichste Variante. Zwei ältere GP fügen dagegen an, dass (c) im Unterschied zu (b) grammatisch korrekter und vollständiger und überdies besonders für den Sprachgebrauch erwachsener Personen (für die sie Sprecher und Angesprochenen damit halten) charakteristisch sei. Mit dieser Begründung geben sie (c) als natürlichste Variante an.

\section{$4 \quad$ Fazit}

Die vorliegende Untersuchung zeigt, dass die Partikel go in allen untersuchten Formulierungen mit den Modalverben chünä, mü̈̈gä, törfä, müäs ä, sölä und welä gleichermassen auf das Verb 'gehen' verweist. In Einzelfällen allerdings scheint go auch andere Funktionen übernehmen zu können (z. B. als Anzeige des sich Zurechtrückens bzw. Anschickens zu einer Tätigkeit). Diese erlauben jedoch aufgrund ihrer geringen Zahl und isolierten Nennungen keine Generalisierungen und wären zudem in einer grösser angelegten Untersuchung auf ihre Akzeptanz hin zu überprüfen.

Bei der feineren Abgrenzung zwischen den beiden Varianten mit go bereitet eine Verallgemeinerung der gewonnenen Resultate hingegen bereits einige Schwierigkeiten. Die erhobenen Daten zeigen nämlich, dass diese Varianten generell sehr unterschiedlich wahrgenommen werden können. Was jedoch mehrfach erwähnt wird, ist die Abstufung der drei Varianten in zeitlicher und/oder räumlicher Dimension: Während Konstruktion (a) darauf verweise, dass die durch den Infinitiv ausgedrückte Tätigkeit unmittelbar und/oder an der Stelle, an der sich Sprecher oder Angesprochener gerade befinden, ausgeübt werden könne, sei bei der Formulierung (b) bis zur auszuführenden Tätigkeit eine mittlere, bei der Variante (c) jedoch eine grössere zeitliche und/oder räumliche Distanz zu überwinden. Dies wirkt sich direkt auf die Akzeptanz der verschiedenen Varianten in den gegebenen Situationen aus: Werden Distanzen (in räumlicher und/oder zeitlicher Hinsicht) als gross empfunden, ist tendenziell auch die Akzeptanz der Variante (c) hoch. Werden solche Distanzen jedoch eher als klein betrachtet 
oder sind sogar entsprechende Hinweise auf geringe Distanzen im Kontext gegeben (z. B. grad in der 5. Aufgabe), wird (c) tendenziell weniger häufig akzeptiert. Diese Beobachtung wäre in einer grösser angelegten Untersuchung auf ihre Relevanz in tatsächlichen Sprechsituationen zu überprüfen.

Eine weitere auffällige Beobachtung ist die unterschiedliche syntaktische Akzeptanz der Variante (c). Während sämtliche älteren GP (c) in allen Aufgaben, wenn auch nicht immer als zur Situation passend, so doch zumindest als syntaktisch möglich oder sogar korrekter als (b) empfinden, lehnen jüngere GP (c) oft aus syntaktischen Gründen ab. Tabelle 9 fasst diese Beobachtung zusammen: ${ }^{12}$ Diese unterschiedliche syntaktische Akzeptanz könnte auf eine grundsätzlich divergierende Interpretation der Variante (c), die sich auch lautlich äussert, zurückzuführen sein.

\begin{tabular}{|l|r|r|}
\hline & jüngere GP & ältere GP \\
\hline $\begin{array}{l}\text { Angaben von (c) als syntaktisch } \\
\text { mögliche Variante (Anz.) }\end{array}$ & $46(76,7 \%)$ & $60(100 \%)$ \\
\hline
\end{tabular}

Tab. 9: Syntaktische Akzeptanz der Variante (c) in den beiden Altersgruppen

So wird die Variante (c) von den älteren GP als Modalverb + gä + Infinitiv + guu, von den jüngeren GP hingegen als Modalverb + gu (oder go) + Infinitiv + guu (oder goo) realisiert. Entsprechend wird auch Variante (b) geäussert, wobei der Infinitiv guu (oder goo) am Ende weggelassen wird. Die bei den älteren GP klare lautliche Differenzierung von gä und guu legt die Vermutung nahe, dass gä und guu auch tatsächlich als zwei verschiedene Lexeme wahrgenommen werden, nämlich, wie Lötscher (1993) in seinem Aufsatz über die Entwicklung der Verbverdoppelung zeigt, als aus der ursprünglichen Nominalpräposition gen entstandene Verbalpräposition ( $g \ddot{a})$ und als Infinitiv $(g u u)$. Die von Lötscher (1993) als weitere Entwicklung postulierte Uminterpretation der ursprünglichen Präposition zum Verb trifft allerdings für gä wahrscheinlich nur teilweise zu, da es sich lautlich immer noch klar vom Infinitiv guu unterscheidet. Gestützt wird diese Behauptung durch die Tatsache, dass gä im untersuchten Dialekt auch als Nominalpräposition noch existiert und v. a. von den älteren Generationen in ihrer ursprünglichen Bedeutung 'nach', 'in Richtung von' weiterhin verwendet wird. ${ }^{13}$ Bei den jüngeren GP hingegen scheint sich die von Lötscher (1993) postulierte Uminterpretation vollständig durchgesetzt zu haben. Dies legt die grosse lautliche Ähnlichkeit von gu und guu nahe. Ausserdem kennen zwar die jüngeren GP die Präposition gä noch in der Bedeutung 'nach', 'in Richtung von', beurteilen sie aber als für ihre eigene Sprache ungebräuchlich. ${ }^{14}$ Zusammenfassend könnte man die unterschiedlichen syntaktischen Interpretationen von (c) für die beiden Altersgruppen folgendermassen darstellen:

i. Ältere GP: Modalverb + Verbalpräposition/Infinitiv des Verbs gehen $(g \ddot{a})+$ Infinitiv + Infinitiv des Verbs gehen ( $g u u)$

ii. Jüngere GP: Modalverb + Infinitiv des Verbs gehen $(g u / g o)$ + Infinitiv + Infinitiv des Verbs gehen (guu/goo)

Anhand dieser Strukturen liesse sich folgendermassen argumentieren: Bei Betrachtung von (ii) wird klar, warum die jüngeren GP die Variante (c) öfter ablehnen und goo als störend empfinden. Da go bereits unmissverständlich als Infinitiv des Verbs gehen interpretiert wird, bedeutet die Verwendung von goo schlicht eine unnötige Doppelsetzung. Die älteren GP

\footnotetext{
12 Zwölf Aufgaben, die von je fünf GP gelöst werden, ergeben pro Altersgruppe 60 Möglichkeiten, eine Variante als syntaktisch möglich oder unmöglich zu beurteilen.

13 Ich stütze mich hierbei auf meine eigenen Beobachtungen des untersuchten Dialekts, die ausserdem auf Rückfrage von drei älteren GP bestätigt wurden.

14 Dies wurde von vier jüngeren GP behauptet und deckt sich mit meinen eigenen Beobachtungen.
} 
hingegen empfinden goo nicht als störend, was darauf zurückzuführen ist, dass in ihrer Interpretation der syntaktischen Struktur der Variante (c) keine offensichtliche Doppelsetzung des Verbs 'gehen' vorliegt, da gä nicht eindeutig und ausschliesslich als Infinitiv, sondern auch bis zu einem gewissen Grad als Präposition empfunden wird. Damit lässt sich die hundertprozentige Akzeptanz von (c) durch die älteren GP erklären. Ausserdem können anhand von Struktur (i) auch die Nennungen, nach denen die Varianten mit go nicht auf das Standarddeutsche 'gehen' zu verweisen scheinen, sondern ähnlich wie die Nominalpräposition gä 'nach', 'in Richtung von' eine Richtung oder ein Ziel vorgeben, besser verstanden werden. Dank der Annahme einer Zwischenform, die sowohl als Infinitiv als auch als Präposition empfunden wird, erscheint überdies auch die häufig gemachte Bemerkung der älteren GP, Konstruktion (b) schliesse (trotz des fehlenden Infinitivs guu) ein Gehen mit ein, nicht als widersprüchlich. Wahrscheinlich lässt sich im Fall von gä also nur "schwer entscheiden, ob man das eine oder andere Wort oder eben eine nicht mehr lösbare Mischung vor sich hat" (Id. $2,324)$.

Was der eben dargestellte Ansatz hingegen nicht erklären kann, ist die Tatsache, dass trotz der für jüngere GP in vielen Fällen störenden Doppelsetzung von 'gehen' in (c) die syntaktische Akzeptanz dieser Variante mit 76,7 \% immer noch hoch ist. Dies muss jedoch nicht zwingend als Schwachpunkt des Erklärungsansatzes gedeutet werden, sondern kann wohl folgendermassen begründet werden: Variante (c) wird, wie die Resultate der Untersuchung nahe legen, im untersuchten Dialekt verwendet und deswegen auch von den jüngeren GP in vielen Fällen als in ihrem Dialekt syntaktisch mögliche Variante angegeben, was allerdings nicht automatisch bedeutet, dass sie diese Variante selbst auch verwenden. ${ }^{15}$ Die dargestellten Überlegungen zu diesem Problem geben Anstoss zu weiterführenden Untersuchungen zu diesem Thema.

Als in Bezug auf die Syntax wenig aussagekräftig erweist sich die Frage nach der natürlichsten Variante. Die Angaben der natürlichsten Varianten sind in der vorliegenden Untersuchung nämlich aufgrund der überwiegenden syntaktischen Akzeptanz aller Varianten vorwiegend durch semantische oder durch eine Mischung semantischer und syntaktischer Kriterien bedingt und werden aufgrund subjektiver Beurteilungen der Situationsbeschreibung oder zusätzlich zum gegebenen Kontext getroffener Annahmen gemacht. In einer zukünftigen Untersuchung wäre es deshalb wünschenswert, der Unterscheidung zwischen semantischen und syntaktischen Kriterien bereits mit einer entsprechenden Anlage des Fragebogens Rechnung zu tragen.

\section{Literatur}

Baur, Arthur (2002): Schwyzertü̈̈tsch. Praktische Sprachlehre des Schweizerdeutschen für Kurse und den Selbstunterricht. 12. Aufl. Winterthur.

Brinkmann, Hennig (1962): Die Deutsche Sprache: Gestalt und Leistung. Düsseldorf.

Bucheli, Claudia/Glaser, Elvira (2002): "The Syntactic Atlas of Swiss German Dialects: Empirical and Methodological Problems". In: Barbiers, Sief/Cornips, Leonie/van der Kleij, Susanne (eds.): Syntactic Microvariation. Amsterdam: 41-74. http://www.meertens.nl/books/synmic/index.html, Stand Sept. 2009.

Christen, Helen (1990): "Neueste Gebrauchsweisen von Modalverben im Schweizerdeutschen". In: Schupp, Volker (ed.): Alemannisch in der Regio. Beiträge zur 10. Arbeitstagung alemannischer Dialektologen in Freiburg/Breisgau 1990. Göppingen: 139-146.

Cornips, Leonie/Poletto, Cecilia (2005): "On Standardising Syntactic Elicitation Techniques". Lingua 115: 939-957.

Eisenberg, Peter (2004): Grundriss der deutschen Grammatik. 2., überarb. und aktual. Aufl. Band II: Der Satz. Stuttgart.

\footnotetext{
15 Vgl. Fussnote 10.
} 
Fischer, Ludwig (1960): Luzerndeutsche Grammatik. Zürich.

Glaser, Elvira (2000): "Erhebungsmethoden dialektaler Syntax". In: Stellmacher, Dieter (ed.): Dialektologie zwischen Tradition und Neuansätzen. Beiträge der internationalen Dialektologentagung, Göttingen, 19.-21. Oktober 1998. Stuttgart: 258-276.

Glaser, Elvira (2003): "Schweizerdeutsche Syntax: Phänomene und Entwicklungen". In: Dittli, Beat/Häcki Buhofer, Annelies/Haas, Walter (eds.): Gömmer MiGro? Freiburg: 3966.

Helbig, Gerhard/Schenkel, Wolfgang (1983): Wörterbuch zur Valenz und Distribution deutscher Verben. 7., unveränd. Aufl. Tübingen.

Id. (1881ff.): Schweizerisches Idiotikon. Wörterbuch der schweizerdeutschen Sprache. Frauenfeld.

Lötscher, Andreas (1993): "Zur Genese der Verbverdopplung bei gaa, choo, laa, aafaa im Schweizerdeutschen". In: Abraham, Werner/Bayer, Josef (eds.): Dialektsyntax. Opladen: 180-200.

Senti, Alois (1988): Anekdoten, Schwänke und Witze aus dem Sarganserland. Mels.

\section{Anhang: Fragebogen}

\section{Anweisungen zum Ausfüllen}

Versetzen Sie sich bitte in die folgenden Situationen 1.-12. Welche der folgenden Varianten a., b. und c. kann man in Ihrem Dialekt in der betreffenden Situation sagen? Welches ist die natürlichste Variante, a., b. oder c.? Würden Sie den Satz normalerweise in einer anderen Form sagen?

N. B.: go wurde als neutralste Form gewählt, kann aber auch für $g u, g \ddot{a}, g i$ etc. stehen.

1. Der kleine Martin sitzt am Boden und spielt mit seiner elektrischen Eisenbahn. Da kommt Jakob dazu, der mit seiner Mutter zu Besuch ist. Er setzt sich zu Martin auf den Boden, denn er will auch mit der Eisenbahn spielen. Er fragt deshalb seine Mutter:

a. Törf i mit em Martin spiilä?

b. Törf i mit em Martin go spiilä?

c. Törf i mit em Martin go spiilä goo?

In der gegebenen Situation mögliche Variante(n):

Natürlichste Variante:

Kommentar/Weitere Variante:

2. Albert ruft Marianne an und fragt, ob sie an diesem Tag schon etwas vorhabe. Er sei nicht zu Hause und seine Frau sei den ganzen Tag allein mit ihren fünf Kindern und wäre froh um etwas Unterstützung. Albert fragt deshalb:

a. Chasch ihrä nid ä chli hälfä?

b. Chasch ihrä nid ä chli go hälfä?

c. Chasch ihrä nid ä chli go hälfä goo?

In der gegebenen Situation mögliche Variante(n):

Natürlichste Variante:

Kommentar/Weitere Variante:

3. Kathrin liegt auf dem Sofa und will ein Nickerchen machen. Just in diesem Moment tritt Pirmin in die Stube, setzt sich zu Kathrin aufs Sofa und fragt, ob sie mit ihm auf den Jahrmarkt fahren will. Da antwortet die müde Kathrin:

a. Wetsch nid au zwerscht ä Moment schloufä?

b. Wetsch nid au zwerscht ä Moment go schloufä?

c. Wetsch nid au zwerscht ä Moment go schloufä goo?

In der gegebenen Situation mögliche Variante(n): 
Natürlichste Variante:

Kommentar/Weitere Variante:

4. Maurus stellt einen frisch gebackenen Kuchen auf den Tisch und will ihn sofort anschneiden. Er bietet den ihn umstehenden Partygästen an, ein Stück zu versuchen:
a. Mügen er au ä Stugg probiärä?
b. Mügen er au ä Stugg go probiärä?
c. Mügen er au ä Stugg go probiärä goo?

In der gegebenen Situation mögliche Variante(n):

Natürlichste Variante:

Kommentar/Weitere Variante:

5. Susanne spielt gerade im Wohnzimmer mit ihren Puppen, als ihr Vater sie auffordert, ihrer Mutter beim Blumengiessen zu helfen. Da antwortet Susanne, die eigentlich lieber weiterspielen will:

a. Muäs i das grad jetz machä?

b. Muäs i das grad jetz go machä?

c. Muäs i das grad jetz go machä goo?

In der gegebenen Situation mögliche Variante(n):

Natürlichste Variante:

Kommentar/Weitere Variante:

6. Jürg sitzt auf dem Sofa und sieht fern. Da kommt seine Mutter ins Zimmer, drückt ihm ein Buch in die Hand und fordert ihn auf, das erste Kapitel zu lesen. Da fragt Jürg unwillig:

a. Muäs i das grad jetz machä?

b. Muäs i das grad jetz go machä?

c. Muäs i das grad jetz go machä goo?

In der gegebenen Situation mögliche Variante(n):

Natürlichste Variante:

Kommentar/Weitere Variante:

7. Anna sitzt in ein Buch vertieft auf dem Sofa. Ab und zu lacht sie laut auf. Albin wundert sich und fragt, was so lustig sei. Da antwortet Anna:

a. Ich ha grad ä paar lustigi Witz gläse. Söl i einä vorläsä?

b. Ich ha grad ä paar lustigi Witz gläse. Söl i einä go vorläsä?

c. Ich ha grad ä paar lustigi Witz gläse. Söl i einä go vorläsä goo?

In der gegebenen Situation mögliche Variante(n):

Natürlichste Variante:

Kommentar/Weitere Variante:

8. Die kleine Caroline hilft ihrer Mutter beim Kochen. Sie möchte aber lieber mit den andern Kindern draussen spielen. Sie fragt deshalb:

a. Mami, törf i nid mit dä anderä Chind spiilä?

b. Mami, törf i nid mit dä anderä Chind go spiilä?

c. Mami, törf i nid mit dä anderä Chind go spiilä goo?

In der gegebenen Situation mögliche Variante(n):

Natürlichste Variante:

Kommentar/Weitere Variante:

9. Es ist nachts um zehn. Vater und Mutter sitzen im Wohnzimmer, als sie plötzlich lautes Weinen aus dem Kinderzimmer hören. Als das Weinen auch nach einigen Minuten nicht verstummt, fragt der Vater:

a. Sölli dä Chind ä Gschichtli vorläsä?

b. Sölli dä Chind ä Gschichtli go vorläsä? 
c. Sölli dä Chind ä Gschichtli go vorläsä goo?

In der gegebenen Situation mögliche Variante(n):

Natürlichste Variante:

Kommentar/Weitere Variante:

10. Es ist fünf vor zwölf und Maria bereitet gerade das Essen zu. Ihr Sohn Daniel steht untätig neben ihr. Da ruft Manuela von der Küchentür aus Daniel zu:

b. Chasch dim Mami nid schnell go hälfä?

b. Chasch dim Mami nid schnell go hälfä?

c. Chasch dim Mami nid schnell go hälfä goo?

In der gegebenen Situation mögliche Variante(n):

Natürlichste Variante:

Kommentar/Weitere Variante:

11. Max und Ernst sind auf einer Party. Max amüsiert sich prächtig und möchte noch eine Weile bleiben. Ernst hingegen ist müde und möchte nach Hause gehen. Er fragt:

a. Wettsch nid au lieber schloufä?

b. Wettsch nid au lieber go schloufä?

c. Wettsch nid au lieber go schloufä goo?

In der gegebenen Situation mögliche Variante(n):

Natürlichste Variante:

Kommentar/Weitere Variante:

12. Es ist Samstagnachmittag. Johanna steht im Garten und grilliert Würste. Währenddessen bereiten Alfred und die Kinder in der Küche die Salate zu. Da fragt Alfred die Kinder:

a. Mügen er nid ä Wurscht probiärä bim Mami?

b. Mügen er nid ä Wurscht go probiärä bim Mami?

c. Mügen er nid ä Wurscht go probiärä goo bim Mami?

In der gegebenen Situation mögliche Variante(n):

Natürlichste Variante:

Kommentar/Weitere Variante: 